\title{
On-line adjustment of battery schedules for supporting LV distribution network operation
}

Conference or Workshop Item

Accepted Version

Zangs, M. J., Yunusov, T., Holderbaum, W. and Potter, B. (2016) On-line adjustment of battery schedules for supporting LV distribution network operation. In: Energy and Sustainability Conference (IESC), 2016 International, 30 Jun -1 Jul 2016, Koln, Germany. Available at https://centaur.reading.ac.uk/67952/

It is advisable to refer to the publisher's version if you intend to cite from the work. See Guidance on citing.

Published version at: http://dx.doi.org/10.1109/IESC.2016.7569485

All outputs in CentAUR are protected by Intellectual Property Rights law, including copyright law. Copyright and IPR is retained by the creators or other copyright holders. Terms and conditions for use of this material are defined in the End User Agreement.

www.reading.ac.uk/centaur 
Central Archive at the University of Reading

Reading's research outputs online 


\title{
On-Line Adjustment of Battery Schedules for Supporting LV Distribution Network Operation
}

\author{
Maximilian J. Zangs*, Timur Yunusov, William Holderbaum and Ben Potter \\ Energy Research Laboratory, School of Systems Engineering \\ University of Reading, Reading, Berkshire, United Kingdom \\ *corresponding author: m.j.zangs@pgr.reading.ac.uk
}

\begin{abstract}
Increasing domestic demand for electric energy is expected to put significant strain on the existing power distribution networks. In order to delay or prevent costly network reinforcement, some UK Distribution Network Operators (DNOs) are investigating the use of Battery Energy Storage Solutions (BESS), or other demand response systems, in the Low-Voltage (LV) power distribution networks to reduce peak demand. In most cases the control strategies, and metrics of success, are evaluated on a half-hourly basis and so sub-half-hourly (i.e. minute by minute) variations in demand are not effectively addressed.

In this work, a closed-loop optimisation methodology is proposed that adjusts the pre-scheduled charging profile of a BESS in a sub-half-hourly manner in order to improve network operation whilst maintain the same average net energy flow over the half-hour period. This new approach guarantees that the BESS follows its predetermined half-hourly schedule, yet voltage and power imbalance, network losses, and feeder overloading are additionally mitigated through sub-half-hourly control actions. For validation, this paper presents a case study based on the real BESS installed in Bracknell as part of Thames Valley Vision project with Scottish and Southern Energy Power Distribution (SSE-PD) evaluated on the IEEE LV test case feeder model.
\end{abstract}

\section{INTRODUCTION}

The ever growing demand for electric energy will place additional strain on the current UK power delivery system. This increase in demand is based on the UK's sociopolitical ambition to reduce emissions, resulting in general electrification of consumer devices, an increased number of renewable energy installations as well as an uptake of electric vehicles. Predictions estimate that by 2030 annual consumer demand will have increased by more than 20TWh [1]. Additionally, penetration of Distributed Energy Resources (DER) will result in higher energy variability, which in turn leads to higher power demand peaks. In fact, the same projection estimates a maximum power flow exceeding 66GW by 2030 .

UK power distribution networks, particularly the LowVoltage (LV) feeders, are under strict regulations to guarantee voltage security and power quality. Violation of these regulations is penalised by the regulatory body, i.e. ofgem in the UK [2], [3]. From a technical aspect, overstressed LV feeders experience higher transmission losses in the best case. Issues such as tripping reclosers or switching feeder branches to less loaded substations may cause temporary power outages, but does not impact the network infrastructure [4], [5]. Continuous line or transformer overloads does lead to equipment fatigue, degradation and eventually failure [6], [7].
In order to cater for future power demand, yet delay costly network reinforcements, recent research has proposed solutions to mitigate high-level power flow issues [8]. To minimise the impact of uncontrolled EV charging, congestion avoidance algorithms have been proposed using, e.g. smoothed particle hydrodynamics [9], online fuzzy coordination strategies [10] or even Nash based game theory [11]. Instead of managing controllable loads, DERs management algorithms have also been proposed. For instance, a robust dispatch optimisation for a hybrid power network that consists of wind, PV, hydro, and thermal power systems has been developed by Peng et.al. [12]. Rather than just focusing on power flow, other studies also considered voltage droop and neutral voltage rise in their DER control [13].

High-level studies optimise power flow and device operation using half-hourly time slots, as this is the standard trading period in the energy exchange. On the other hand, high frequency grid support to uphold power quality by maximising the power factor, reducing harmonic currents and balancing phases has also been proposed. Singh et.al. proposed an improved linear sinusoidal tracer to allow better current injection for harmonic compensation [14]. LV power can also be upheld by improving the power factor, and this was done without exciting harmonic resonance [15].

Managing power flow and power quality on a half-hourly period or at very high frequencies does not, however, cater for power flow issues on a minute to minute basis. Subhalf-hourly power spikes are averaged in the half-hourly power demand profiles, yet are the most impacting events for Distribution Network Operators (DNOs), since these spikes are significantly higher than the average power flow. Recently, Scottish and Southern Energy - Power Distribution (SSEPD) has deployed several Energy Storage Management Units (ESMU) in south England to investigate the impact of Battery Energy Storage Solutions (BESS) on these sub-half-hourly power events [16]. Since DNOs need to schedule their device operation, an effective strategy to control the ESMUs has to be developed.

In this work a sub-half-hourly closed-loop optimisation methodology is presented that controls BESS operation in such a way that the average half-hourly schedules are followed, yet minute-by-minute demand variability is additionally addressed. For this paper, the half-hourly schedule is assumed to have been previously determined and is provided as an 
TABLE I: Cost Function Parameters

\begin{tabular}{|c|c|}
\hline$s_{\text {loss }}(t)$ & Network's apparent power losses where \\
\hline$j_{1}{ }^{\prime} \quad l \quad(t)$ & $\begin{array}{l}p_{\text {loss }}(t)+j q_{\text {loss }}(t)=\mathbf{s}_{\text {loss }}(t) \in \mathbb{R}_{\geq 0}^{T} \\
\text { Current in all lines where }\end{array}$ \\
\hline$\imath_{\text {line }, l, p}(t)$ & {$\left[i_{\text {line }, 1,1}(t), \ldots, i_{\text {line }, L, 3}(t)\right]=\mathbf{i}_{\text {line }}(t) \in \mathbb{R}^{T \times L \times 3}$} \\
\hline$v_{l o a d, i}(t)$ & $\begin{array}{l}\text { Single phase voltages at all customers where } \\
{\left[v_{\text {load }, 1}(t), \ldots, v_{\text {load }, I}(t)\right]=\mathbf{v}_{\text {load }}(t) \in \mathbb{R}^{T \times I}}\end{array}$ \\
\hline$s_{s s, p}(t)$ & $\begin{array}{l}\text { Apparent power vector measured at substation where } \\
{\left[s_{s s, 1}(t), s_{s s, 2}(t), s_{s s, 3}(t)\right]=\mathbf{s}_{s s}(t) \in \mathbb{C}^{T \times 3}}\end{array}$ \\
\hline$i_{s s, p}(t)$ & $\begin{array}{l}\text { Complex current vector measured at substation where } \\
{\left[i_{s s, 1}(t), i_{s s, 2}(t), i_{s s, 3}(t)\right]=\mathbf{i}_{s s}(t) \in \mathbb{C}^{T \times 3}}\end{array}$ \\
\hline$v_{\text {bess }, p}(t)$ & $\begin{array}{l}\text { Phase-to-neutral voltage vector measured at BESS where } \\
{\left[v_{\text {bess }, 1}(t), v_{\text {bess }, 2}(t), v_{\text {bess }, 3}(t)\right]=\mathbf{v}_{\text {bess }}(t) \in \mathbb{R}^{T \times 3}}\end{array}$ \\
\hline
\end{tabular}

input. There are a range of methods presented in the literature to create such half-hourly schedules that generate schedules based on historical data and peak reduction algorithms [17]. This half-hourly schedule is then a constraint within the closed-loop optimisation methodology.

The closed-loop optimisation methodology updates the minute-by-minute power output values for the BESS based on historical power values and a model of the feeder. In order to determine the next power value for the BESS, the model of the feeder is repetitively simulated within the optimisation procedure to determine the power value for the BESS that best improves the operation of the feeder in terms of a specific set of metrics such as bus voltages, power flow, phase imbalance and losses. A range of different cost functions, based on these metrics, are evaluated and compared in this paper. The consideration of sub-half-hourly demand variations, whilst maintaining the expect half-hourly operation, in order to improve network performance is a novel contribution to the field.

\section{Methodology}

In order to improve the LV network's operation on a sub-half-hourly period, the prescheduled power profile of a grid-connected BESS is dynamically adjusted. Here, network operation is indicated by several simulation outputs, that become cost function parameters, which are then fed into their corresponding cost functions. Minimising these cost functions results in improved network operation. The simulation determines the network states at a given instant in time. In some cases the cost function parameter is an array of values for the whole feeder; e.g. $\mathbf{v}_{\text {load,i }}(t)$ contains the voltages at all customer nodes on the feeder.

All of the used cost function parameters are tabulated in Table I and are split into two groups. The first group captures the entire network's performance and are obtained from simulations, and the second group represents realistic measurements that may be obtained during deployment. For example, for the BESS installed in the Thames Valley Vision project there is access to substation monitoring of power and current per phase, and phase-to-neutral voltages and BESS energy consumption at the BESS. Detail on these cost functions and how they are used is explained next.

\section{A. Cost Functions}

In total, ten cost functions $\left(\zeta_{n}\right.$ where $\left.n=1, \ldots, 10\right)$ were defined. The first three costs functions $\left(\zeta_{1}\right.$ to $\left.\zeta_{3}\right)$ are based on complete system information, i.e. full observability with $\mathbf{s}_{\text {loss }}(t), \mathbf{i}_{\text {line }}(t)$, and $\mathbf{v}_{\text {load }}(t)$, and the seven remaining cost functions $\left(\zeta_{4}\right.$ to $\left.\zeta_{10}\right)$ are based on realistic network measurements, i.e. partial observability with $\mathbf{s}_{s s}(t), \mathbf{i}_{s s}(t)$, and $\mathbf{v}_{\text {bess }}(t)$.

1) Network losses: Without expensive measuring equipment it is impossible to accurately estimate network losses, yet the minimisation of them can result in better network operation. From simulations in OpenDSS, apparent power losses are recorded in a vector $\mathbf{s}_{\text {loss }}(t)$. The complete network losses over the entire day can be computed from $s_{\text {loss }, l} \in \mathbf{s}_{\text {loss }}$.

$$
\zeta_{1}\left(\mathbf{s}_{\text {loss }}\right):=\sum_{t=1}^{T}\left|s_{\text {loss }}(t)\right|
$$

2) Customer voltage deviation: In the UK, network voltages are bound to an operational band of $240 \mathrm{~V}+10 \%-6 \%$. Violation of these regulatory bands is penalised and fined. A convex cost that minimises at the network's nominal operating voltage, $V_{n}$, is defined below. The same function maximises as voltage level deviates towards the lower voltage threshold, $V_{l}$, or higher voltage threshold, $V_{h}$. The cost is based on all customers' (or loads') voltages, $\mathbf{v}_{\text {load }}(t)$. This voltage vector contains the voltage profile for load $i=\{1, \ldots, I\}$ at time slot $t$, where $v_{\text {load }, i}(t) \in \mathbf{v}_{\text {load }}(t)$. For any time slot this cost, $\zeta_{2}$, identifies the worst voltage deviation recorded throughout the entire network. It is defined as follows (here $v_{l o a d, i}(t)$ is shortened to $v_{i}$, and $\mathbf{v}_{\text {bess }}(t)$ is shortened to $\mathbf{v}$ ):

$$
\zeta_{2}(\mathbf{v}):=\max _{i} \begin{cases}\zeta^{h}\left(v_{i}\right) & \text { if } V_{n} \leq v_{i} \leq V_{h} \& \zeta^{h}\left(v_{i}\right)<\zeta^{\max } \\ \zeta^{l}\left(v_{i}\right) & \text { if } V_{l} \leq v_{i}<V_{n} \& \zeta^{l}\left(v_{i}\right)<\zeta^{\max } \\ \zeta^{\max } & \text { otherwise }\end{cases}
$$

Here, $\zeta^{\max }$ is introduced to limit the maximum cost and prevent any runaway behaviour. Furthermore, voltage deviation towards voltages higher than nominal voltage, $V_{n}$, is captured in the sub-cost $\zeta^{h}(v)$, and the cost for voltage levels falling below $V_{n}$ is captured by $\zeta^{l}(v)$.

$$
\begin{aligned}
\zeta^{h}(v) & :=\left|\left(\frac{v-V_{n}}{V_{h}-V_{n}}\right)^{2}-1\right|^{-1} \\
\zeta^{l}(v) & :=\left|\left(\frac{V_{n}-v}{V_{n}-V_{l}}\right)^{2}-1\right|^{-1}
\end{aligned}
$$

3) Feeder utilisation: Preventing excessive feeder utilisation to minimise the quadratic line losses and also avoid equipment damage is the aim of minimising the $\operatorname{cost} \zeta_{3}$. Here, the vector of all line currents, $\mathbf{i}_{\text {line }}(t)$, is used. From this, the maximum cost is found for a specific $i_{\text {line }, l, p}(t) \in \mathbf{i}_{\text {line }}(t)$ for any given moment in time, $t$. The cost function itself is defined as follows:

$$
\zeta_{3}\left(\mathbf{i}_{\text {line }}(t)\right):=\max _{l}\left(\sum_{p=1}^{3}\left(\frac{i_{\text {line }, l, p}(t)}{i_{\text {rated }, l}}\right)^{2}\right)
$$


Here, the ratio between the phase currents $i_{l i n e, l, p}$ where $p=\{1,2,3\}$ and the line's phase rating $i_{\text {rating, }}$ is squared to simulate the quadratic relationship between line current and thermal losses.

4) Voltage deviation at BESS: Similar to Equation 2, the voltage deviation measured at the BESS is used here. This is the first cost based on partial observability (here $v_{\text {bess }, p}(t)$ is shortened to $v_{p}$, and $\mathbf{v}_{\text {bess }}(t)$ is shortened to $\mathbf{v}$ ):

$$
\zeta_{4}(\mathbf{v}):=\max _{p} \begin{cases}\zeta^{h}\left(v_{p}\right) & \text { if } V_{n} \leq v_{p} \leq V_{h} \& \zeta^{h}\left(v_{p}\right)<\zeta^{\max } \\ \zeta^{l}\left(v_{p}\right) & \text { if } V_{l} \leq v_{p}<V_{n} \& \zeta^{l}\left(v_{p}\right)<\zeta^{\max } \\ \zeta^{\max } & \text { otherwise }\end{cases}
$$

5) Voltage imbalance at BESS: Different phase loadings cause asymmetric currents which lead to imbalanced network voltages. Since imbalanced three phase systems operate suboptimally in comparison to balanced networks, a cost that captures this imbalance is defined as follows:

$$
\zeta_{5}\left(\mathbf{v}_{\text {bess }}(t)\right):=\max _{p}\left(\frac{\left|\mathbf{v}_{\text {bess }}(t)-\overline{\mathbf{v}}_{\text {bess }}(t)\right|}{\overline{\mathbf{v}}_{\text {bess }}(t)}\right)
$$

Here, $\overline{\mathbf{v}}_{\text {bess }}$ denotes the mean phase voltage, computed form the phase to neutral voltage vector $\mathbf{v}_{\text {bess }}$. The cost itself is defined as the maximum voltage divergence from the mean, divided by the mean.

6) Active power imbalance at substation: From substation monitoring, the apparent power vector $\mathbf{s}_{s s}(t)$ can be monitored. This vector contains three complex powers, where each real component represents the phase's active load and the imaginary component represents the phase's reactive load.

$$
\zeta_{6}\left(\mathbf{s}_{s s}(t)\right):=\max _{p}\left(\frac{\left|\operatorname{Re}\left(\mathbf{s}_{s s}(t)\right)-\operatorname{Re}\left(\overline{\mathbf{s}}_{s s}(t)\right)\right|}{\operatorname{Re}\left(\overline{\mathbf{s}}_{s s}(t)\right)}\right)
$$

Balancing the apparent power itself might lead to unwanted phase angle distortion, since excess reactive power might be injected. Therefore only the real component, i.e. active power, is included in the imbalance calculation for this cost.

7) Reactive power at substation: The reactive power component is defined as another cost, $\zeta_{7}$, since its minimisation would free line capacity without impacting the BESS's state of charge.

$$
\zeta_{7}\left(\mathbf{s}_{s s}(t)\right):=\sum_{p}^{3}\left|\operatorname{Im}\left(s_{s s, p}(t)\right)\right|
$$

8) Neutral current at substation: Feeder lines in the UK are often asymmetric in shape. Commonly, the three phase cores are significantly larger in cross-section than the neutral line. Therefore, any neutral current is expected to result in higher network losses. Hence, the following cost is defined as the magnitude of this neutral current:

$$
\zeta_{8}\left(\mathbf{i}_{s s}(t)\right):=\left|\sum_{p=1}^{3} i_{s s, p}(t)\right|
$$

The neutral current is calculated by summing the complex phase currents, $i_{p}(t)$ where $p=\{1,2,3\}$.

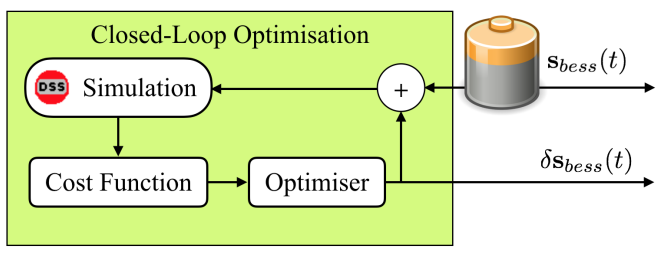

Fig. 1: BESS Power Optimisation

9) Power factor at substation: The amount of "useful" power transmitted over the network is captured by the power factor (PF). Maximising this value at 1 implies that no reactive power is consumed. Since reactive power can easily be compensated for by the BESS without impacting its battery schedule, the cost $\zeta_{9}$ is defined as the difference between the maximum and actual PFs per phase.

$$
\zeta_{9}\left(\mathbf{s}_{s s}(t)\right):=3-\sum_{p=1}^{3} \frac{\operatorname{Re}\left(s_{s s, p}(t)\right)}{\left|s_{s s, p}(t)\right|}
$$

Here, PF for each phase is calculated as the fraction of the phases active power and the magnitude of the apparent power. Aggregating all phase's PFs would result in a maximum value of 3 , therefore any difference from this value is defined as the cost.

10) Feeder loading at substation: Transformers in substations are rated sufficiently high to cater for the power demand of an individual feeder. Yet the feeding cables on the other hand might reach capacity limitations much sooner. Therefore, the final cost is defined as the sum of all phase capacities.

$$
\zeta_{10}\left(\mathbf{i}_{s s}(t)\right):=\sum_{p=1}^{3}\left(\frac{i_{s s, p}(t)}{i_{\text {rated }}}\right)^{2}
$$

Here, the ratio between the phase currents $i_{s s, p}$ where $p=$ $\{1,2,3\}$ and its substation's phase rating $i_{\text {rating }}$ is squared to simulate the quadratic relationship between line current and thermal losses.

\section{B. Optimisation and Simulation}

The underlying procedure that performs the closed-loop optimisation is captured in Figure 1. Here, for each time slot, $t$, a pre-scheduled BESS power value, $\mathbf{s}_{b e s s}(t)$, is extracted and adjusted by an offset vector, $\delta \mathbf{s}_{\text {bess }}(t)$. This offset vector is found through an optimiser that minimises a selected cost function by repetitively running simulations of the distribution feeder. To avoid impacting the schedule of the BESS, a constraint is defined so that the internal battery's dis/charging power remains the same:

$$
0=\sum_{p}^{3} \operatorname{Re}\left(\delta s_{b e s s, p}(t)\right) \forall t
$$

Additionally, two more constraints are defined to limit the BESS's maximum power flow. This restriction is due to the power electronic's and battery rating's limitations. 


$$
\begin{aligned}
S_{p e} & \geq\left|s_{\text {bess }, p}(t)\right| \text { where } p=\{1,2,3\} \forall t \\
S_{\text {battery }} & \geq \sum_{p}^{3} \operatorname{Re}\left(s_{\text {bess }, p}\right) \forall t
\end{aligned}
$$

Here, $S_{p e}$ reflects the maximum per-phase apparent power of the BESS and $S_{\text {battery }}$ is the nominal rating of the battery. After having found an optimal $\delta \mathbf{s}_{\text {bess }}(t)$, the adjusted BESS power, i.e. $\mathbf{s}_{\text {bess }}(t)+\delta \mathbf{s}_{\text {bess }}(t)$, is fed into the simulation once again, and all outputs from this snapshot are saved. Then closed-loop optimisation processes is then repeated for the next time step $(t+1)$ until a complete day has been simulated.

\section{Evaluation}

The saved outputs are analysed in order to evaluate the network's performance when minimising a particular cost function for BESS power optimisation. This analysis is performed by feeding the simulation outputs that resulted from optimisation based on full observability (i.e. minimising $\zeta_{1}$, $\zeta_{2}$ or $\zeta_{3}$ ) into the cost functions based partial observability (i.e. $\zeta_{4}$ to $\left.\zeta_{10}\right)$. Similarly, this sensitivity analysis was also reversed to asses the impact of minimising costs $\zeta_{4}$ to $\zeta_{10}$ on costs $\zeta_{1}$ to $\zeta_{3}$.

\section{Case Study}

Five case studies were assessed, where each used one of the first three cost functions to optimise the entire network's operation. The network used was the UK based IEEE LV test case network as published on the PES website [18]. From the accompanying power profiles ( 1 minute in period), the combination that generates the highest power spike of $68 \mathrm{~kW}$ was found and applied to the simulations. This spike occurred around 9:30am (i.e. $t=566$ minutes). Location of the BESS was determined by using previous work on finding optimal placement for installation of BESS in LV networks [19].

The BESS power was adjusted using a Sequential Quadratic Programming (SQP) optimiser, and consisted of two independent mechanisms: a reactive power and active power component. Two baselines were initially obtained, where the first did not include and BESS operation and the second consisted of applying a traditional or "normal" half-hourly BESS schedule. Three variations of the control mechanism were assessed next, where either active power, reactive power or apparent power was adjusted on a minute-by-minute basis. These five studies can be summarised as follows:

1 Base case without any BESS operation

2 Half-hourly, i.e. traditional BESS operation

3 Reactive power only; $0=\operatorname{Re}\left(s_{\text {bess }, p}\right) \forall p$

4 Active power only; $0=\operatorname{Im}\left(s_{\text {bess }, p}\right) \forall p$

5 Both active and reactive power within constraints

\section{RESUlts}

In this section an excerpt to show a sub-half-hourly optimised BESS operation to reduce network losses is presented
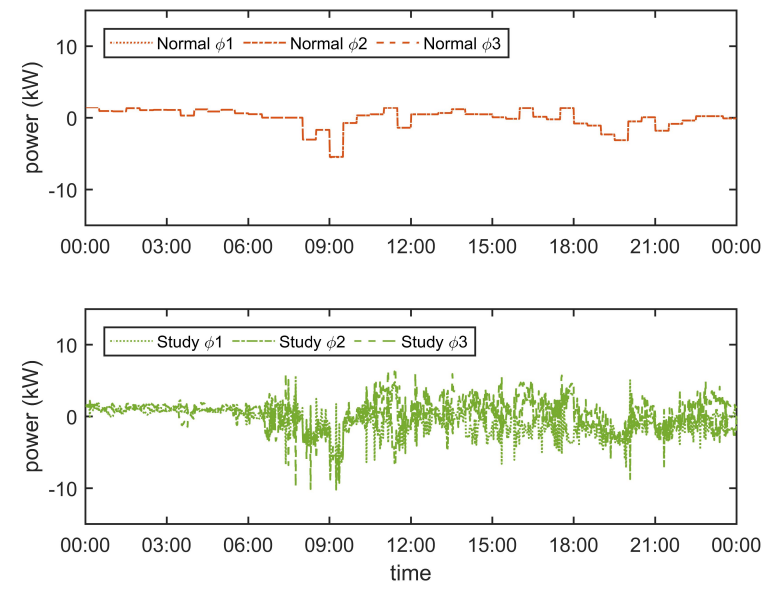

Fig. 2: BESS powers per phase for normal half-hourly operation (top) and adjusted operation (bottom).
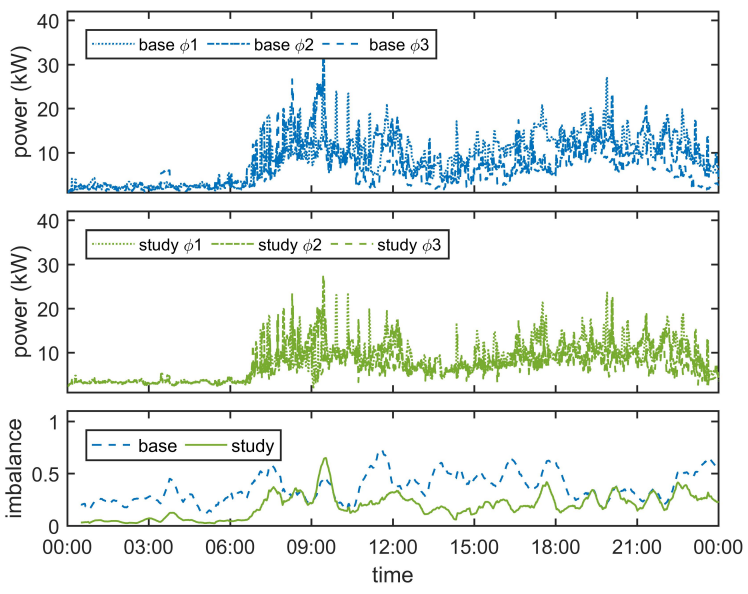

Fig. 3: Phase imbalance measured at substation for baseline operation (top) and adjusted BESS operation (bottom).

first. Following these results is the aforementioned crosssensitivity analysis. This analysis evaluates the sensitivity of $\zeta_{4}$ to $\zeta_{10}$ based on the minimisation of costs $\zeta_{1}$ to $\zeta_{3}$ and vice versa.

\section{A. Network Improvements from Loss Minimisation}

For this excerpt, the network losses, $\zeta_{1}$, were minimised for each time step. In the following figures, the resulting BESS profiles, power and voltage imbalances, and distribution losses are plotted against time. Here, the performance for adjusting both active and reactive power, i.e. Study 5 (hereon referred to as the "study") are compared against the baseline and traditional BESS operation results.

In Figure 2, both the traditional BESS phase powers and the adjusted phase powers have been plotted over time. It can be observed that for the traditional operation mode, the BESS divides its scheduled power value equally over all three phases. In contrast, for the study results, all three phases are 
TABLE II: Mininmising Network Losses
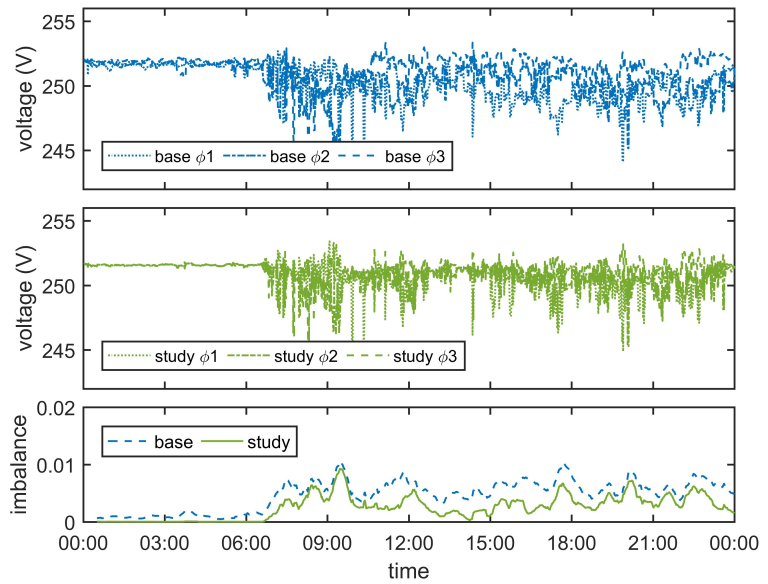

Fig. 4: Voltage imbalance measured at BESS bus for baseline operation (top) and adjusted BESS operation (bottom).

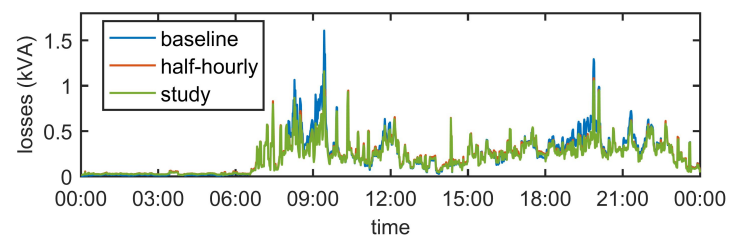

Fig. 5: Network losses measured during the day for baseline system operation, traditional BESS operation, and improved BESS operation.

used independently after having been optimised on a sub-halfhourly basis.

The impact of applying these adjusted power values becomes apparent when investigating the improvements they causes on the network. A reduction in phase imbalance is one of the improvements and is shown in Figure 3. Here, the three phase active powers, measured at the network's substation, have been plotted for both the baseline and the study results. Additionally, the imbalance index for both has been included to quantify the improvement.

Voltage deviations, as measured at the BESS bus, have also been reduced. This is shown in Figure 4, where the voltage discrepancy has been effectively mitigated in study. Similar to the power imbalance plot, the voltage imbalance has also been included in Figure 4, where improvements can be observed over the entire day.

Finally, the reduction in network losses is presented. When operating the BESS in its traditional mode a reduction in network losses from 5.66kVAh per day to $5.32 \mathrm{kVAh}$ per day can be observed. With the study using the optimised BESS powers, the network losses were reduced even further to $5.06 \mathrm{kVAh}$ per day. This comparison is plotted in Figure 5.

\section{B. Cross-Sensitivity of Costs}

Next, the sensitivity of minimising $\zeta_{1}$ to $\zeta_{3}$ on $\zeta_{4}$ to $\zeta_{10}$ is analysed. This was done by separately minimising $\zeta_{1}$ to $\zeta_{3}$,

\begin{tabular}{r|ccccccc} 
Study & $\zeta_{4}$ & $\zeta_{5}$ & $\zeta_{6}$ & $\zeta_{7}$ & $\zeta_{8}$ & $\zeta_{9}$ & $\zeta_{10}$ \\
\hline 2 & 0.933 & 0.999 & 0.960 & 1.001 & 1.000 & 0.941 & 0.960 \\
3 & 0.807 & 0.794 & 0.962 & 0.611 & 0.722 & 0.556 & 0.982 \\
4 & 0.359 & 0.261 & 0.342 & 1.002 & 0.397 & 0.971 & 0.955 \\
5 & 0.653 & 0.534 & 0.508 & 0.794 & 0.607 & 0.782 & 0.965
\end{tabular}

TABLE III: Minimising Load Voltage Deviation

\begin{tabular}{r|ccccccc} 
Study & $\zeta_{4}$ & $\zeta_{5}$ & $\zeta_{6}$ & $\zeta_{7}$ & $\zeta_{8}$ & $\zeta_{9}$ & $\zeta_{10}$ \\
\hline 2 & 0.933 & 0.999 & 0.960 & 1.001 & 1.000 & 0.941 & 0.960 \\
3 & 0.543 & 0.962 & 0.951 & 2.987 & 1.107 & 6.544 & 0.471 \\
4 & 0.723 & 0.822 & 0.792 & 1.002 & 0.738 & 1.025 & 0.955 \\
5 & 0.512 & 0.872 & 0.955 & 2.788 & 0.889 & 6.052 & 0.527
\end{tabular}

TABLE IV: Minimising Feeder Utilisation

\begin{tabular}{r|ccccccc} 
Study & $\zeta_{4}$ & $\zeta_{5}$ & $\zeta_{6}$ & $\zeta_{7}$ & $\zeta_{8}$ & $\zeta_{9}$ & $\zeta_{10}$ \\
\hline 2 & 0.933 & 0.999 & 0.960 & 1.001 & 1.000 & 0.941 & 0.960 \\
3 & 1.099 & 1.005 & 0.945 & 4.737 & 1.009 & 9.747 & 0.195 \\
4 & 5.925 & 3.325 & 1.754 & 1.002 & 2.941 & 7.791 & 0.686 \\
5 & 3.266 & 2.243 & 1.731 & 4.842 & 2.034 & 10.891 & 0.164
\end{tabular}

TABLE V: Network Loss Sensitivity

\begin{tabular}{r|ccccccc} 
Study & $\zeta_{4}$ & $\zeta_{5}$ & $\zeta_{6}$ & $\zeta_{7}$ & $\zeta_{8}$ & $\zeta_{9}$ & $\zeta_{10}$ \\
\hline 2 & 0.937 & 0.937 & 0.937 & 0.937 & 0.937 & 0.937 & 0.937 \\
3 & 1.893 & 1.260 & 2.086 & 0.934 & 1.204 & 0.933 & 2.498 \\
4 & 0.909 & 0.864 & 1.044 & 0.964 & 0.913 & 0.864 & 2.119 \\
5 & 1.835 & 1.276 & 1.559 & 0.967 & 1.333 & 0.954 & 3.045
\end{tabular}

TABLE VI: Customer Voltage Sensitivity

\begin{tabular}{r|ccccccc} 
Study & $\zeta_{4}$ & $\zeta_{5}$ & $\zeta_{6}$ & $\zeta_{7}$ & $\zeta_{8}$ & $\zeta_{9}$ & $\zeta_{10}$ \\
\hline 2 & 0.999 & 0.999 & 0.999 & 0.999 & 0.999 & 0.999 & 0.999 \\
3 & 0.498 & 0.633 & 2.502 & 1.456 & 0.591 & 1.228 & 0.992 \\
4 & 0.419 & 0.350 & 1.127 & 1.178 & 0.459 & 0.427 & 4.003 \\
5 & 0.332 & 0.425 & 1.427 & 1.745 & 0.481 & 1.270 & 2.464
\end{tabular}

TABLE VII: Feeder Utilisation Sensitivity

\begin{tabular}{r|ccccccc} 
Study & $\zeta_{4}$ & $\zeta_{5}$ & $\zeta_{6}$ & $\zeta_{7}$ & $\zeta_{8}$ & $\zeta_{9}$ & $\zeta_{10}$ \\
\hline 2 & 0.960 & 0.960 & 0.960 & 0.960 & 0.960 & 0.960 & 0.960 \\
3 & 0.433 & 0.798 & 0.476 & 0.982 & 0.834 & 0.990 & 0.195 \\
4 & 0.950 & 0.952 & 0.848 & 0.956 & 0.946 & 0.956 & 0.686 \\
5 & 0.421 & 0.795 & 0.672 & 0.971 & 0.816 & 0.983 & 0.164
\end{tabular}

following the procedure explained in Section II-B for each of the five case studies and computing $\zeta_{4}$ to $\zeta_{10}$ from the resulting outputs.

Table II shows the resulting $\zeta_{4}$ to $\zeta_{10}$ for the minimisation of $\zeta_{1}$, i.e. network losses. Similarly, Table III shows the resulting $\zeta_{4}$ to $\zeta_{10}$ for the minimisation of $\zeta_{2}$, i.e. voltage deviation of all customers, and Table IV presents the resulting $\zeta_{4}$ to $\zeta_{10}$ for the optimisation in respect to $\zeta_{3}$, i.e. feeder utilisation.

In this analysis, the 1st study acted as the baseline, was used to normalise all resulting costs, and has been removed from the table as all values would be equal to 1 . For the remaining studies, a value below 1 indicates a reduction of the associated cost when optimising based on the corresponding $\zeta$. In contrast a value above 1 reflects an increase in the associated cost.

Tables V, VI, and VII present the sensitivity analysis in the opposite direction. Here, $\zeta_{4}$ to $\zeta_{10}$ were minimised and their impacts on the overall network performance captured by 
$\zeta_{1}$ to $\zeta_{3}$ are tabulated.

The first set of tables shows the impact of optimising the BESS operation based on complete network information. In contrast the last three tables show the impact on the entire network when optimising the BESS powers based on realistically limited network information. Similar to before, a value below 1 indicates an improvement in network operation in respect to the baseline, whereas a larger value implies worsening the corresponding cost.

\section{DISCUSSION}

From the excerpt results that capture the time-series improvements, it was demonstrated that optimising the BESS operation in sub-half-hourly time steps yielded visible improvements to the network operation. Nonetheless, the proposed methodology has limited adjustment flexibility when the pre-scheduled profiles make the BESS operate close to its power electronic's limitations. This becomes particularly apparent in the morning (see Fig. 2-5 around 9:30am), when the highest power peak of the day is compensated, and does not leave much room for additional power adjustments. In fact, the power imbalance was worsened during this time. Despite the phase power adjustments, the BESS operated as planned and thus guarantees to inject and absorb the predetermined powers, whilst also providing vital grid support.

The sensitivity analysis shows that active power adjustments, as performed in Study 4, are generally the most beneficial. Controlling only reactive power, as done in Study 3 , or both active and reactive power, as done in Study 5, did not provide the same level of cross-cost minimisation. Alternatively, the reason for Study 4 outperforming the other two BESS operation modes may be due to the underlying distribution network being more resistive than inductive by nature. Therefore active power adjustments yield higher impact than the reactive power component would.

\section{CONCLUSION}

In this work, a closed-loop optimisation methodology to control BESS operation was presented. This methodology adjusts BESS power on a minute-by-minute by incorporating a feeder simulation into an SQP optimiser based on ten different cost functions. The simulation outputs that determined the operational performance of the feeder were fed as input parameters into cost functions which were minimised by the optimiser. These cost function parameters either consisted of measurements throughout the entire network, resulting in best optimisation, or a reduced number of parameters to replicate realistic deployment limitations and constraints on observability.

Being able to dynamically adjust BESS operation on a sub-half-hourly basis, whilst following a predetermined power profile enables improved performance compared to existing approaches that only consider half-hourly control actions. For example, network losses were reduced by double the amount in comparison to traditional or half-hourly BESS operation. By extending BESS control systems to respond to sub-half-hourly demand variations enables such system to perform better, and so strengthens the business case for their use to support the network and postpone conventional network reinforcement.

\section{ACKNOWLEDGMENT}

The authors would like to thank IT Networking Group at the University of Reading for providing the HTCondor compute cluster, and also Miss C. Scrivener for proofreading this manuscript.

\section{REFERENCES}

[1] National Grid, "Future Energy Scenarios 2015," Tech. Rep. July, 2015

[2] Health and Safety Executive, "Electricity Safety, Quality and Continuity Regulations," 2002.

[3] Consumer Focus, "Guaranteed Stdandard of Electricity Distribution," 2010 .

[4] D. T. Nguyen, "Modeling Load Uncertainty in Distribution Network Monitoring," IEEE Transactions on Power Systems, vol. 30, no. 5, pp. 2321-2328, 2015

[5] M. a. Eltawil and Z. Zhao, "Grid-connected photovoltaic power systems: Technical and potential problemsA review," Renewable and Sustainable Energy Reviews, vol. 14, no. 1, pp. 112-129, jan 2010.

[6] S. Weckx and J. Driesen, "Load Balancing With EV Chargers and PV Inverters in Unbalanced Distribution Grids," IEEE Transactions on Sustainable Energy, vol. 6, no. 2, pp. 635-643, 2015.

[7] M. W. Siti, D. V. Nicolae, A. a. Jimoh, and A. Ukil, "Reconfiguration and load balancing in the LV and MV distribution networks for optimal performance," IEEE Transactions on Power Delivery, vol. 22, no. 4, pp. 2534-2540, 2007.

[8] D. E. Olivares, A. Mehrizi-Sani, A. H. Etemadi, C. a. Cañizares, R. Iravani, M. Kazerani, A. H. Hajimiragha, O. Gomis-Bellmunt, M. Saeedifard, R. Palma-Behnke, G. a. Jiménez-Estévez, and N. D. Hatziargyriou, "Trends in microgrid control," IEEE Transactions on Smart Grid, vol. 5, no. 4, pp. 1905-1919, 2014.

[9] Q. R. Hamid and J. A. Barria, "Congestion Avoidance for Recharging Electric Vehicles Using Smoothed Particle Hydrodynamics," IEEE Transactions on Smart Grid, vol. 31, no. 2, pp. 1014-1024, 2016.

[10] A. S. Masoum, S. Deilami, and M. A. S. Masoum, "Fuzzy Approach for Online Coordination of Plug-In Electric Vehicle Charging in Smart Grid," IEEE Transactions on Sustainable Energy, vol. 6, no. 3, pp. 1112$1121,2015$.

[11] N. Z. Xu and C. Y. Chung, "Challenges in Future Competition of Electric Vehicle Charging Management and Solutions," IEEE Transactions on Smart Grid, vol. 6, no. 3, pp. 1323-1331, 2015.

[12] C. Peng, P. Xie, L. Pan, and R. Yu, "Flexible Robust Optimization Dispatch for Hybrid Wind/Photovoltaic/Hydro/Thermal Power System," IEEE Transactions on Smart Grid, vol. 7, no. 2, pp. 751-762, 2016.

[13] M. J. E. Alam, K. M. Muttaqi, and D. Sutanto, "Community Energy Storage for Neutral Voltage Rise Mitigation in Four-Wire Multigrounded LV Feeders With Unbalanced Solar PV Allocation," IEEE Transactions on Smart Grid, vol. 6, no. 6, pp. 2845-2855, 2015.

[14] B. Singh, C. Jain, and S. Goel, "ILST control algorithm of single-stage dual purpose grid connected solar PV system," IEEE Transactions on Power Electronics, vol. 29, no. 10, pp. 5347-5357, 2014.

[15] S. Lin, D. Salles, L. Cui, and Q. Liu, "A Novel Control Method for Power Factor Compensation on Harmonic Distorted Low Voltage Power Systems," Harmonics and Quality of Power (ICHQP), 2012 IEEE 15th International Conference on, vol. 3, no. 3, pp. 76-83, sep 2012.

[16] Scottish and Southern Energy Power Distribution, DNV-GL, GE, Honeywell, L. Fund, University of Reading, and Bracknell Forest Council, "Thames Valley Vision - Project library - Published documents," 2016.

[17] M. Rowe, T. Yunusov, S. Haben, C. Singleton, W. Holderbaum, and B. Potter, "A Peak Reduction Scheduling Algorithm for Storage Devices on the Low Voltage Network," IEEE Transactions on Smart Grid, vol. 5, no. 4, pp. 2115-2124, 2014.

[18] IEEE PES Distribution System Analysis Subcommittee, "Distribution Test Feeder Working Group," 2016.

[19] T. Yunusov, D. Frame, W. Holderbaum, and B. Potter, "The impact of location and type on the performance of low-voltage network connected battery energy storage systems," Applied Energy, vol. 165, pp. 202-213, 2016. 